\title{
Temps et lieu dans le théâtre Tudor : deux traitements remarquables
}

\section{André Lascombes}

\section{(2) OpenEdition}

\section{Journals}

Édition électronique

URL : http://journals.openedition.org/shakespeare/511

DOI : 10.4000/shakespeare.511

ISSN : 2271-6424

Éditeur

Société Française Shakespeare

Édition imprimée

Date de publication : 1 novembre 1989

Pagination : 23-42

\section{Référence électronique}

André Lascombes, "Temps et lieu dans le théâtre Tudor : deux traitements remarquables », Actes des congrès de la Société française Shakespeare [En ligne], 6| 1989, mis en ligne le 01 janvier 2007, consulté le 01 mai 2019. URL : http://journals.openedition.org/shakespeare/511 ; DOI : 10.4000/ shakespeare.511 


\section{SOCIETE FRANCAISE SHAKESPEARE}

\section{Actes du Congrès - 1984}

\section{LIEU ET TEMPS}

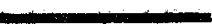

Directeur de la publication Jean FUZIER 


\section{Temps et lieu dans le théâtre Tudor : Deux traitements remarquables}

On doit à Anne Righter une étude qui, bien que déjà ancienne, propose un point de vue toujours utile sur certains aspects de ce que l'on peut nommer la crise du théâtre Tudor ${ }^{1}$. Cette crise est un processus d'adaptation à une véritable révolution culturelle qui bouscule, plus encore que les institutions, les modes de vie et les pensées, et qui s'accélère depuis les dernières décennies du XVe siècle jusqu'au delà de ce que l'on appelle "Renaissance ".

L'un des retentissements marquants de cette crise dans l'ordre du théâtre affecte la relation qu'il établit avec son auditoire. Mais au-delà même de cette modification profonde, point de départ de la thèse d'A. Righter, il y a un élément clé qui change sensiblement dans le fonctionnement théâtral : la nature du lien référentiel unissant le signe théâtral à ce qu'il entend représenter.

A l'encontre de ce qu'indiquent souvent les sémiologues spécialistes $d u$ théâtre moderne, le signe théâtral issu de la scène médiévale anglaise, et tout particulièrement celle des Cycles, renvoie, outre qu'à lui-même, à un référent ultime de nature spécifique : il est non pas historique mais métaphysique ${ }^{2}$. L'ampleur de l'écart ainsi créé 
entre les deux pôles du lien référentiel suscite chez qui contemple ce théâtre (qui jamais ne cesse d'être jeu, mais qui est aussi, on le sait bien, transport rituel vers des ailleurs mythiques) un phénomène de flou dans lequel se télescopent le plan du surréel mythique figuré et celui des formes humaines ou phénoménales représentées. J'ai choisi d'appeler ce flou référentiel diaphore théâtrale ${ }^{3}$.

Il est toutefois légitime de dire que ce phénomène affecte, avec une ampleur et des effets très différents, toute autre forme de théâtre, puisque celui-ci naît toujours si je puis dire de ce double regard porté sur le signe scénique.

Ce bref rappel, qui donne à comprendre la nature des difficultés que doit surmonter pour s'adapter le théâtre du premier âge Tudor, explique aussi que son évolution n'affecte pas seulement la relation qu'il entretient avec ses auditoires, mais la nature même des éléments sur lesquels il opère. A l'inverse, il souligne un aspect majeur de la permanence de la forme théâtrale qui requiert toujours du spectateur, même si c'est par des modalités diverses, la même constante aptitude à user du double regard.

La présente étude entend se limiter à l'examen de deux formalisations théâtrales du lieu et du temps dramatiques, intéressantes dans la mesure où elles sont des expérimentations un peu anomales et à ce titre trop souvent négligées, probablement suscitées par l'adaptation de la praxis théâtrale poursuivie au long de la période Tudor.

Si les conclusions de cette enquête sur deux formes exceptionnelles ne sont pas directement transposables à l'étude de formes plus moyennes et peut-être mieux adaptées à l'usage du temps, elles livrent pourtant des enseignements utiles. On gardera à l'esprit, au long de l'examen, trois questions corrélées, même si la seconde seule est l'objet de l'étude :

- comment ces formes procèdent de la modification référentielle que l'on a rappelée, et comment elles y répondent.

- quelles stratégies théâtrales elles mettent en œuvre pour réaliser temps et lieu scéniques.

- de quelles valeurs ou principes esthétiques procèdent les solutions qu'elles privilégient. 
Un mot d'explication paraît encore nécessaire, en présentant cette réflexion au Congrès de la Société Française Shakespeare. Le choix de la pièce d'H. Medwall (1497) précède de quelque 60 années le terminus a quo de ce qui est le champ d'études de cette Société. Cette outrageuse violation de la règle est délibérée mais peut s'expliquer, sinon s'excuser. Il paraissait important de soumettre à comparaison deux pièces qui, situées presque aux deux bouts d'une première phase de mutations théâtrales et toutes deux exceptionnelles, comme on l'a dit, mettent en œuvre d'une part deux projets nettement contradictoires en regard du temps et du lieu, et d'autre part, semblent par ces procédures opposées exprimer le même souci de réactiver en termes nouveaux le fonctionnement de la diaphore médiévale dont le souvenir s'éternise au long de la période et qui inspire encore partiellement la relation à la scène shakespearienne.

A - L'espace-temps et le ressourcement de la diaphore : "Fulgens and Lucres ", (1497) ou la boulimie théâtrale.

C'est à partir des prémisses de l'analyse de Mrs Righter que j'aborde cette pièce décrite par l'auteur comme " a godely interlude", et sans doute jouée en 1497 à Lambeth Palace, résidence de John Morton, Archevêque de Canterbury, au cours d'un banquet réunissant, autour des ambassadeurs de Flandre et d'Espagne en négociations à Londres, des courtisans et la maisonnée du Cardinal.

\section{Les structures du jeu :}

L'argument explicite, reflétant les stratégies politiques d'Henry VII, fait la démonstration exemplaire que la vraie noblesse réside dans le mérite et la vertu de la personne plutôt que dans son lignage. Mais l'intérêt théâtral de la pièce, au delà de cet aspect discursif, découle plutôt de la greffe diégétique et spectaculaire que constitue l'immixion dans la pièce de deux personnages prétendument issus de l'auditoire et qui établissent avec lui et avec l'intrigue un rapport de double connivence.

La décision d'introduire cet augment, sommairement jugée par A. Righter comme soumission condamnable à la "tyrannie d'un auditoire ${ }^{4}$ qui exige d'être maintenu dans la situation de connivence ou d'inclusion au jeu héritée du " théâtre médiéval rituel ", doit s'apprécier, me semble-t-il, de façon beaucoup plus nuancée. Outre que le 
respect des goûts de l'auditoire n'est pas nécessairement une erreur pour un théâtre qui demeure en son âge vernaculaire, la décision d'instituer des personnages médiatiques à la lisière de l'aire du jeu et de l'aire des spectateurs revient à offrir un nouveau champ spatiotemporel au déploiement dramatique et théâtral et, avec cette nouvelle dimension référentielle, à restituer au regard théâtral sa richesse diaphorisante.

Je propose de recenser brièvement sous trois rubriques les effets que me semble autoriser (sinon avoir de fait assuré en cette occasion) cette utilisation spatio-temporelle du jeu. Ces effets sont liés à la triple fonction que remplit l'acteur à deux têtes (nommées dans la pièce $A$ et $B$ ) en se plaçant à la jonction du jeu et du hors-jeu dramatiques. Acteur-médiateur d'une part, il est en même temps, si l'on autorise ce pléonasme barbare, acteur agonique inséré dans la diégèse, et enfin acteur dramaturge en ce qu'il participe activement avec le spectateur à la création dramatique et théâtrale. Si l'on ne peut décrire le contenu de ces trois fonctions que de façon consécutive, il convient de souligner qu'elles opèrent de fait simultanément, et en synergie totale lors du déroulement du spectacle.

1. 1. L'acteur-médiateur et l'espace-temps du spectacle

G. Wickham, dont on connaît la contribution au renouvellement des perspectives critiques sur le théâtre Tudor et l'expérience de praticien du théâtre, résume d'une formule efficace ce premier effet, voyant dans ce choix structurel "a device perfectly calculated to foreshorten time and place and admirably realised to secure just that effect ${ }^{5}$. En y voyant encore l'équivalent de l'effet qu'assurait dans le théâtre des Cycles la manipulation référentielle de l'espace-temps du récit dramatique, à savoir le télescopage des deux référents et l'établissement garanti de l'effet de diaphore, l'auteur fait à HenryMedwall, dont Richard Southern rappelle opportunément par ailleurs quels liens étroits il entretient avec le monde du théâtre, un compliment flatteur. Son stratagème, en raccourcissant la distance optique et psychologique entre jeu et spectateur, entraîne le même intéressement complice qu'éprouvait le spectateur médiéval devant les sbires d'Hérode poignardant les Innocents dans les rues de sa propre ville, ou à voir ses propres voisins dénoncer Marie dans le Cycle de la Ville de $N$. Il est intéressant de suivre le processus qui, 
dès le début de la pièce, embraye pour ainsi dire le temps vécu du spectateur sur celui de la fiction, et anastomose dans le hall festif l'aire où festoient les dîneurs à celle du jeu.

S'adressant à l'auditoire (vers 1 à 27) en un discours qui a aussi valeur de didascalie, $\mathrm{A}$ le décrit en train de ripailler. Par là, il l'officialise irrécusablement dans sa situation spectatrice tout en l'élevant, contradictoirement et sur le mode badin, au rang d'objet de spectacle : l'auditoire se contemple en ce discours.

Pour confirmer les virtualités de l'ambiguïté ainsi créée, A et B en suscitent aussitôt une autre (vers 28-63), sur leur propre identité cette fois : ces spectateurs présumés, qui nient être des acteurs, n'en résument pas moins la pièce dont le début est imminent, et, au nom des mœurs de leur propre temps, en contestent les conclusions. C'est là une nouvelle façon de souligner le rapport de la fiction à l'actualité et pour tout dire sa proximité à l'auditoire. Identifiant les acteurs qui entrent enfin - Fulgens, et bientôt Lucrèce - ils cherchent à se fondre dans l'auditoire craignant d'être expulsés du " hall ". Dans cet ultime rôle de spectateurs égarés et naïfs qu'affole la proximité du spectacle, ils oublient l'élémentaire distinction entre le comédien et l'acteur dans le débat qui s'offre à eux. Leur ultime démarche avant de disparaître est donc de rappeler la réalité têtue de l'auditoire d'une part, et d'autre part le règne désormais irrécusable d'une fiction reconnue et qui, bien qu'attendue comme telle, s'impose comme forme rivale du réel quotidien.

Dans ce rôle d'entremetteur spectaculaire, l'actant médiateur, représenté par ces deux personnages complémentaires du faux naïf, cherche en entraînant les spectateurs à sa suite dans la zone intermédiaire, suscitée puis niée, à amplifier en eux les deux attitudes contraires et complémentaires qui font l'auditoire : la participation et la contemplation distanciée. Intensifiant en lui la duplicité du regard, il décuple l'émotion contradictoire que tire le spectateur de la stance théâtrale.

Avant de le ramener à la primauté du réel vécu, par le débrayage progressif et inverse des deux univers, réalisé en fin de première partie, les deux compères vont au long des quelque 1200 vers qui restent entretenir dans l'auditoire cette superposition d'attitudes, ce regard diaphorisant. 


\section{2. L'acteur agonique et la complexité des espace-temps}

La fiction une fois mise en route par l'arrivée de Fulgens le Sénateur, et de Cornelius le patricien qui prétend à la main de la belle Lucrèce, nos spectateurs-commentateurs, un instant oubliés, ressurgissent. Cornélius vient d'exprimer sa perplexité dans l'un de ces vocatifs directs, hérités de la pratique médiévale, que l'acteur jette vers l'auditoire. Il cherche un serviteur avisé qui l'aide en son entreprise amoureuse. B, immédiatement séduit malgré l'effroi de A qui le rappelle à sa condition de spectateur, court après Cornelius faire ses offres de service. Intronisé dans sa nouvelle fonction et bientôt imité par A qui réfléchit tout haut que l'aventure vaut mieux que sa présente condition de chômeur, il est désormais, et A avec lui, l'actif gestionnaire de la fiction. Assurés de la matérielle et de la confiance de leurs maîtres respectifs, les compères vont prendre leurs aises avec la mission d'ambassade dont chacun est tour à tour investi auprès de Lucrèce, en profitant pour avancer leurs affaires auprès de la suivante, Joan, qui a pour eux bien des charmes. Par ce détournement de la fiction, dévoyée un moment à leur profit, sont alimentés les effets de l'embrayage initial et de la participation spectatrice. Dans toute la mesure où la convention d'analogie demeure, en effet, le spectateur adhère à l'occasion qui lui est offerte de vivre par procuration la série d'initiatives parfois drôles, parfois d'une pesante bouffonnerie, que chacun des deux acolytes greffe sur la diagèse initiale. Il s'agit moins ici de crédibilité et de conviction que d'un accord entretenu entre le spectateur et ceux qui se sont d'emblée déclarés être ses porte-parole. Le spectateur, entré dans ce contrat, ratifie par une partie de lui-même l'activité anarchique qui infléchit parfois dangereusement le cours de la fiction proposée, et partage un peu de la griserie, médiocre certes mais entraînante, qu'il y a à se voir abandonner le destin du monde ludique.

Techniquement, cette tacite connivence est entretenue par une suite régulière d'invites, faites directement ou indirectement à l'auditoire, à partager la responsabilité de ce qui se dit et se fait dans l'aire du jeu. Le commentaire que fait B du faste vestimentaire de Cornelius, son maître, dont les goûts sont étrangements proches de ceux, présumables, de l'auditoire, la satire du mariage, celle des femmes ou des hommes battus, sont autant de rengaines du temps ou de clins 
d'œil plus ou moins lourds suscitant les réflexes les moins nobles de l'auditoire pour cautionner les affirmations intervenant dans l'univers du jeu. Il n'y a pas moins d'une douzaine de ces vocatifs, directs ou indirects, adressés au public, pour préserver la convention d'homologie entre son univers et celui dans lequel A et B, depuis peu sortis de ses rangs, exercent leur autorité.

On peut bien sûr juger avec Anne Righter que cette mise en résonance de l'univers du spectateur et de celui du jeu, puisqu'elle ne relève plus d'une idéologie cohérente avec la fable représentée, n'est que l'exercice méprisable d'une convention désormais sans objet. J'apprécierai pour ma part autrement le bricolage spectaculaire d'Henry Medvall, qui va être repris une deuxième fois, de façon plus sobre mais tout aussi magistrale, dans la deuxième partie de la pièce, au terme d'un entracte explicitement voué à satisfaire les appétits impatients d'un auditoire dont la pièce a interrompu le festin.

Le point de vue d'A. Righter revient en effet à décider que le théâtre de la période (et ceci est plusieurs fois suggéré dans son étude) aurait eu pour seule finalité l'intention rituelle ou didactique et ne saurait aucunement prétendre à exercer - ou ce serait très indûment - quelque fonction de divertissement esthétique. Or, les textes, dont celui d'H. Medwall, imposent une autre évidence. La structure diaphorisante suscitée par les cautions référentielles n'entrave en effet nullement, mais tout au contraire encourage, le fonctionnement simultané d'un double jeu, établissant de constantes liaisons entre le registre bouffon (bien à tort dit "réaliste») et la perception, plus ou moins fine d'ailleurs, des implications spirituelles ou didactiques.

Il est donc plus sage de dire que le théâtre, fût-il didactique ou rituel, propose toujours, lorsqu'il est authentique, un plaisir indissociable de ses significations idéologiques ou éthiques. Plaisir grave qui naît de la contemplation, sérieuse plus souvent que bouffonne, d'aspects fondamentaux de notre vérité. Sensible fréquemment dans les Cycles et dans les Moralités, il s'exprime en maint passage de Fulgens and Lucres. Je l'y analyse comme le deuxième effet, et sans doute le plus puissant, du télescopage des deux univers du jeu et du vécu.

Cet effet, il faut sans doute l'attribuer au troisième aspect de la mission qu'exécutent les personnages de la médiation, A et B. 


\section{3. L'acteur dramaturge et le plaisir théâtral}

J'ai peut-être échoué à faire entendre que cet acteur médiateur, qui gère de bout en bout la fiction jouée et l'auditoire, est continûment ou presque le régisseur alerte, omniprésent - sinon omniscient - du processus spectaculaire. Gardant sans cesse un œil sur l'auditoire, et jonglant périlleusement avec un spectacle dont il conserve malaisément tous les fils - pareil en cela au clown dont les maladresses cascadantes attestent l'impeccable maîtrise, il tient sans discontinuer le spectacle à bout de bras. Spectateur du spectateur, mais aussi son serviteur et son maître, l'incitant sans cesse à le suivre au cœur du jeu, il exhausse la fonction spectatrice en ostendant et mettant à portée de l'auditoire tous les constituants du spectacle. Enumérons-les brièvement.

Le médiateur commence par magnifier, pour tous ces ventres qui l'écoutent, l'outrecuidance qu'il y a pour le non-acteur à brûler la frontière, et entrer dans le Jeu. Ce tabou absolu une fois trangressé (mais dans le cadre du jeu), l'activité désordonnée des compères propose au spectateur le plaisir quasi-gustatif et un peu sulfureux qu'il y a à voir anarchiser une structure concertée, et en prévoir les dévergondages. Leur témérité à gérer le spectacle, dénoncée puis niée par les compères dès les vers 359-408, est à nouveau soulignée de multiples fois (vers $359-408$; 574-632; 817-18). Le sentiment prévaut cependant que ces chômeurs aussi ingénieux qu'imprévoyants, lancés dans une entreprise de "théâtre au noir " qu'ils gèrent aux frais de leurs maîtres, courent tous les périls et opèrent tous les miracles. Ils assurent par là l'adhésion, non seulement à l'efficacité de leur gestion, mais aussi à leur amoralisme tranquille qui impose régulièrement pour vérité la feinte et le mensonge. Sans doute l'essentiel de la saveur de cette pièce est-il dans cette ostension célébrée du rôle joué et du masque adopté, auxquels la force de conviction confère instantanément les marques de l'identité.

Ainsi, ayant induit l'assentiment tacite du spectateur en imposant l'identité du vécu avec le temps et le lieu où s'inscrit l'action contemplée, $\mathrm{A}$ et $\mathrm{B}$ nous donnent à déguster toute pure l'essence du plaisir théâtral : la fondamentale labilité de l'être jouant avec les masques que la Fortune lui propose et cette force plasmatrice dont, même provisoirement, il dispose. Dans un registre plus épique, c'est 
de cette séduction-là que participe la force théâtrale de Richard Gloucester, tel que le dessine William Shakespeare.

\section{Appréciation}

A l'évidence, cette médiation militante qui cherche à gagner à la pièce tout l'univers péri-ludique n'a qu'un principe, celui de l'amalgame. C'est sur lui qu'elle construit la participation boulimique que l'on a décrite, qu'elle l'installe scéniquement, théâtralement et même esthétiquement. Un bref aperçu convaincra de cette analogie dans la triple démarche.

2. 1. Pour ce qui est des lieux et des temps physiques sur lesquels s'appuie la réalisation théâtrale de Fulgens and Lucres, reconnaissons que le choix fait par $\mathrm{H}$. Medwall fonctionne admirablement dans le cadre physique de la réalité sociale à laquelle la pièce est destinée, disons le hall aristocratique.

Ce hall, même s'il est spatialement hiérarchisé et s'il admet prioritairement les maîtres de céans et les dignitaires, connaît une promiscuité sociale dont l'époque moderne, qui en a rapidement perdu le goût après la Renaissance, mesure mal les effets encore aujourd'hui. Autour des tables, réservées aux hôtes de marque, s'empresse certainement la nuée de ceux qui vivent dans la mouvance des mấtres. Qu'une éventuelle troupe soit ou non composée de telles gens, les acteurs côtoient certainement les livrées, et ceux des invités qui sont en surnombre à table : s'amassent peut-être dans l'aire médiane et près du screen ${ }^{6}$. Le spectacle, en ce cas, occupe le centre du hall. Qu'il utilise ou non une plateforme basse postée contre le screen face à la table d'honneur, comme le pense Southern, ${ }^{7}$ il demeure autour de ce lieu précis une aire seconde du jeu qui ne se distingue pas formellement de celle laissée à l'auditoire. Même si la fiction jouée devait adopter un temps différent du temps vécu dans le hall (et l'on relève dans Fulgens \& Lucres les traces nettes de la stratégie qui homogénéise les deux temporalités ${ }^{8}$ ), elle partage avec la vie du hall le même espace.

Ce n'est en rien diminuer le mérite d'H. Medwall que de reconnaître que la structure théâtrale de sa pièce n'est que l'élaboration esthétiquement efficace d'une pratique issue tout droit des habitudes de la vie quotidienne. 


\section{2. La diaphore ressourcée}

Cette élaboration consiste en fait à aller un pas plus loin que la tradition, et même à renverser les habitudes. Au lieu que l'auditoire soit tenté de participer par la proximité où il est du spectacle, c'est l'action dramatique qui ici s'installe délibérément sur le territoire des spectateurs au terme d'une annexion qui est conceptuelle autant que spatiale. Dès lors, le spectateur englobe du regard ces deux territoires même si la frontière entre eux, constamment franchie au nom de la nouvelle règle du jeu, n'est jamais tout à fait abolie. Cette stratégie de l'amalgame, soigneusement entretenue tout du long comme on l'a vu, explique aisément le maintien de la diaphore. Sur sa lancée de spectateur médiéval, l'invité présent dans le hall, conservant le double regard qui lisait deux sens en un seul signe, déchiffre sans difficulté la double nature du lieu.

La diaphore acquiert cependant là un sens nouveau. Sa finalité idéologique, ici obsolescente, est remplacée - avec une désinvolture qu'A. Righter a bien notée et qui la peine - par une autre : la vacillation du regard se fait esthétique. Entretenu dans la conscience qu'il est à la fois impliqué dans l'univers clos du jeu et qu'il lui demeure extérieur, le spectateur accède à la vision du théâtre contemplé comme tel par ceux qui participent à son fonctionnement. On note à plusieurs reprises dans la pièce l'émergence indiscutable d'un tel métathéâtre 9 . Il serait aventureux de dire qu'il s'agit là d'une première. La jubilation réflexive sur le jeu en acte est un privilège déjà octroyé au spectateur médiéval et paraît un élément constitutif du plaisir que proposent certaines formes du théâtre dit primitif.

Constatons seulement que Medwall fait un usage brillamment renouvelé d'une catégorie de la saveur théâtrale dont les meilleurs élisabéthains (on pense bien sûr à Kyd et à W. Shakespeare) sauront réexploiter la tradition.

Si la médiation réussit si parfaitement à s'imposer dans Fulgens \& Lucres, ce n'est pas seulement parce que l'y invitent la topographie scénique et l'acquis théâtral de l'auditoire, mais sans doute aussi parce qu'une telle pratique s'harmonise naturellement au climat culturel. Le lecteur de la pièce note non sans quelque surprise que cette pièce offerte à un parterre d'ambassadeurs et de puissants du jour, développe complaisamment des épisodes dont la bouffonnerie, 
lorsqu'elle n'est pas seulement bon enfant, flirte sans ambages avec le graveleux et le scatologique. Le fait qu'il s'agisse d'une pièce de Noël, retrouvant certains aspects de l'esprit d'inversion et de désordre rituel propre à cette période calendaire, renforcerait, plutôt qu'elle ne nuancerait, l'hypothèse que la diégèse greffée, faisant mieux passer ainsi peut-être le caractère potentiellement révolutionnaire et scandaleux des données débattues dans la diégèse primaire, exploite un consensus ou du moins une connivence culturelle à peu près sans faille.

B - GORBODUC : La diérèse de l'espace-temps, et la diaphore « réservée " ${ }^{10}$

Il me semble intéressant de m'arrêter maintenant, et par contraste, sur une solution donnée au traitement du temps et du lieu à l'autre extrémité de la période considérée. Traitement tout aussi remarquable en ce qu'il opte pour une pratique antinomique de la première pour satisfaire, ce sera du moins mon hypothèse, à l'efficacité "théâtrale ". Mes remarques tâcheront d'expliciter ces guillemets.

Il s'agit du traitement que donnent au temps et au lieu dramatiques Th. Norton et Th. Sackville dans leur célèbre co-production Gorboduc jouée comme on le sait, pour la première fois, à l'Inner Temple, en 1561.

Unanimement louée comme une étape importante de l'expression de la tragédie en Angleterre, elle est tout aussi unanimement condamnée pour sa médiocre qualité spectaculaire. Richard Southern, qui verrait volontiers dans cette pièce un exemple intéressant (parce qu'a contrario) de la pragmatique théâtrale dont il cherche (dans son ouvrage The Staging of Plays before Shakespeare,) les étapes, entre les premiers interludes du début du siècle et les "plaisantes tragédies " et "tragiques comédies" des années 1560-1580, renonce à tout examen parce que la formule en est, dit-il, trop différente $^{11}$. C'est, je l'ai dit, la raison même qui m'a fait retenir la pièce.

1. Le traitement du temps et du lieu :

Les critiques le plus communément adressées à Gorboduc concernent le caractère excessivement formaliste de son discours qui 
est d'une rhétorique très judiciaire, enserrée dans le corset des règles et artifices. Bien que ce point de vue soit inattaquable, il est incomplet et ne saurait constituer, s'agissant d'une œuvre dramatique, que la première moitié d'un jugement satisfaisant.

Le recours à une langue peu naturaliste, volontiers soumise aux rites de l'écriture théorique, et d'accent parfois hiératique, ne suffit pas en effet à condamner le dramaturge des premières décennies de la période élisabéthaine, comme en témoigne le cas de Thomas Kyd entre autres. Il peut être plus intéressant par contre de noter, comme le font au moins deux critiques, que le discours tient, non de son architecture propre, mais de l'orientation spatiale qui semble lui être donnée dans l'utilisation dramatique, le caractère plus rhétorique que spectaculaire qu'on lui reproche ${ }^{12}$. Projeté vers le lieu de l'auditoire plutôt qu'adressé par l'acteur à son vis-à-vis dramatique, ce verbe n'est que très rarement celui que supposent l'échange et l'interlocution; il est le plus souvent celui de l'oratio. Et si l'on y regarde de près, on s'aperçoit que cette rigidité ou plutôt cette rigueur systématique de la forme linguistique ont des échos dans le traitement que les auteurs de Gorboduc réservent au temps et à l'espace dramatiques.

\section{1. Le temps}

Facteur éminent du climat et de la conscience tragiques, le temps figure en bonne place dans le discours des divers acteurs du drame. On peut même dire qu'il est l'un des agents essentiels, constituant la donnée élémentaire. Cette histoire exemplaire d'un règne perdu, d'un royaume souillé et saccagé, se fonde en effet sur la notion insistante que l'essence de la fonction royale, héritage, préservation et transmission d'un pouvoir, réside dans la durée. Le roi Gorboduc le souligne avec force d'emblée (1, 2, 80-85). L'évocation de cette durée, temporalité étirée des brumes du passé jusqu'aux grisailles de l'avenir, conduit tous ceux qui pensent royalement dans la pièce à répéter l'éloge de la stabilité, éternité du prince. (Gorboduc 1, 2, 115-145 ; Ferrex, II, 1, 15-21). L'on pourrait même dire à cet égard que la longueur des tirades constitue une traduction iconique de ce thème dans le discours. La même obsession temporelle suscite d'autres thèmes majeurs : 
- le souvenir du passé mythique héroïsé, qui est leçon pour l'avenir (Eubulus, 1, 2, 317-406) ;

- deux motifs alternés et dramatiquement antagonistes, qui sont d'une part celui du fugit tempus (qui résonne dès l'ouverture et revient obsessionnellement dans le discours de Gorboduc), et celui d'autre part de l'urgence tragique, du sentiment que l'occasion qu'offre un présent incertain doit être au plus vite saisie afin d'écarter les menaces toujours plus angoissantes du futur (Hermon, II, I, 81-161 ; Ferrex II, 1 162-193). Le thème du futur nié (dès l'acte II par Gorboduc, Cassandre un peu grotesque, puis par tous à l'acte V) achève de faire triompher cette angoisse.

La technique de présentation elle-même impose cette tension temporelle caractéristique du climat tragique. Ou bien les faits ont déjà eu lieu : décisions prises, crimes perpétrés (mort de Ferrex, III, I, 158-165 ; mort de Porrex IV, 2, 247-258), ou bien ils semblent inévitables. Ce rendu pathétique de l'angoisse et du désespoir nourrit de nombreuses interventions choriques (Doran, II, I, 194-213 ; Philander, II, 2, 280-95; chorus final de l'acte II; pathos angoissé de Videna, I, I et IV, 1, 1-81). Celles-ci assurent un effet soutenu de redondance amplificatrice.

\section{2. Le lieu et l'espace dramatiques}

Cet exemple d'excellente gestion dramatique de l'un des agents importants rend plus impérieuse encore la question de savoir pourquoi est réputée a-théâtrale une pièce qui contient des éléments potentiellement efficaces. Je crois trouver un élément de réponse dans la façon dont est traité dans Gorboduc l'espace dramatique.

On doit à Dieter Mehl, dans le précieux historique qu'il consacre $\mathrm{au}$ "dumb show", ce mimodrame ou tableau aux multiples origines utilisé comme forme dramatique au sein de mainte pièce élisabéthaine et jacobéenne, une stimulante appréciation de la valeur des cinq mimodrames qui ouvrent chacun des cinq actes de la tragédie de Gorboduc $^{13}$. Les remarques qui suivent lui doivent d'avoir considérablement progressé dans leur élaboration. Je les réduirai au bref rappel de l'analyse que propose $\mathrm{D}$. Mehl et à quelques additions de ma part. 
Notant le fait, d'évidence banale, qu'une parfaite césure existe dans la pièce entre texte parlé et mimodrame, notant aussi le caractère, soit allégorique, soit pittoresque et d'un pathos emphatique, de ces tableaux muets, il exprime en conclusion le point de vue que le recours à des scènes sémantiquement redondantes, véhiculant avec une symétrie parfaite un message identique pour l'essentiel mais de formalisation si antithétique, procède d'un choix esthétique plutôt que de l'incompétence, ou du simple respect des modèles académiques. Ce point de vue me paraît fécond et peut trouver quelque confirmation dans une remarque complémentaire qui, si elle n'est pas en soi déterminante, a pourtant valeur d'indice.

La recherche dans le corps du texte écrit de mentions locales ou topographiques qui feraient pendant à l'utilisation appuyée, et que l'on a notée, des références temporelles, conduit à un premier constat intéressant: le texte (celui de Norton surtout) est pratiquement vierge de toute référence spatiale. L'apparition spontanée, dans les tout premiers vers, de la seule évocation suceptible d'être spatialement imaginée, même si elle est prioritairement vouée à transcrire la durée, demeure à peu de chose près une remarquable exception ${ }^{14}$. Par ailleurs, il est difficile d'invoquer à preuve ou à contre preuve le bourgeonnement d'images spatiales dans la partie attribuée à Sackville : le thème du partage et celui de l'éloignement génèrent chez lui diverses belles images, dont la flamboyante teichoscopie retraçant la mort de Porrex ${ }^{15}$. On peut seulement constater que ces occurrences remarquables sont très rares et que cette rareté même fait question. Question qui se nourrit d'autres interrogations encore, comme l'absence à peu près absolue de références au lieu scénique et à la façon dont l'action verbale de chaque acte doit être spatialement réalisée : faut-il imaginer par exemple un seul lieu successivement occupé par les acteurs, parfois très nombreux, qui ouvrent chaque acte avec leur dumb show, puis par les "entre-parleurs"?

On n'est finalement certain que de deux choses: d'une part, de la soigneuse opposition de deux medias scéniques, texte et mimodrame, et de leur qualité d'impact ; d'autre part, du va-et-vient régulièrement entretenu par leur alternance dans le déroulement scénique. Le choix d'un système et même d'une stratégie esthétique paraît incontestable. Il commande donc que l'on essaie d'en apprécier le sens, sinon toutes les raisons. Je me harsarderai donc à examiner plus avant l'hypothèse de D. Mehl. 
2. Essai d'évaluation du fonctionnement théâtral de Gorboduc: théâtre déconstruit ou diaphore "réservée "?

Ma démarche s'appuie sur une brève analyse du fonctionnement spectaculaire de la pièce, telle que la reçoit son auditoire présumé.

\section{1. Réception et diaphore}

Dans ce spectacle rigoureusement circonscrit à un univers fictif dissocié du cadre physique de la vie vécue, le spectateur, s'il n'est. plus appelé aux intrusions que sollicitait efficacement Medwall dans sa pièce, se voit cependant proposer une activité de multiples lectures du signe. Comme en tout drame de vertu théâtrale, il perçoit concurremment :

- les comédiens évoquant le roi Gorboduc, les princes Ferrex et Porrex, etc..

- les personnages historiques qui ont nom "Gorboduc", "Ferrex", "Porrex", etc..., esthétiquement évoqués dans la présente réalisation.

Cette première et double perception, premier effet de diaphore, est suscitée par le seul texte verbal (que l'on appellera la partie B du texte). Notons que les "entreparleurs " qui restituent dans ce texte le rappel mythique, héroïsé, rhétoriquement pathétique parfois, du passé historique anglais sont des présences physiques certes mais qu'ils demeurent, même si l'on pouvait (et à quels signes verbaux ?) les créditer d'une restitution gestuelle du texte, confinés à une activité largement éolienne.

- une troisième lecture est cependant suggérée, mais c'est surtout par le biais des tableaux vivants (que l'on appelle partie A du texte). Ceux-ci imposent le parallélisme entre l'histoire et le tableau emblématique ou allégorique, suggérant que les acteurs historiques, ou les actants qu'ils formalisent, ont un troisième et ultime référent: cet autre drame, inscrit dans le présent ou les menaces du futur, du pays politiquement réel qu'est l'Angleterre de l'auditoire.

Mais, alors que le didactisme médiéval voulait que l'oscillation interprétative du signe théâtral jusqu'au référent ultime théologiquement assigné fût immédiate et inscrite dans le texte verbal ou ses implications, ici - si du moins ma lecture est correcte - la dissociation des signes entre texte verbal et tableau atténue très sensiblement la réalité sémiologique de l'acteur - et derrière lui l'actant - qui 
est seul capable d'implanter le drame dans la réalité présente. A l'inverse de ce qui advient par exemple dans Respublica, l'Angleterre n'est, ici, jamais physiquement réalisée dans le texte B. Son évocation purement linguistique, systématiquement exempte comme on l'a vu de toute marque spatiale, la confine au plan prudemment lointain de concept historique.

2.2. L'efficacité spectaculaire: théâtre déconstruit ou diaphore "réservée"?

$\mathrm{Au}$ terme de ce descriptif, on pourra penser que la dissociation des éléments du texte de la représentation a des effets radicaux sur le fonctionnement diaphorisant, et donc sur l'ampleur et l'intensité des décodages du signe. De fait, il semble bien que l'ensemble de la critique, plus sensible à l'importance prioritaire de l'élément B et du registre rhétorique qui l'informe, ait privilégié l'hypothèse, plus ou moins explicitement formulée, qu'il s'agit là d'un théâtre pauvre, scéniquement misérable. Et la description qui précède paraîtrait bien accréditer la vue d'un théâtre éclaté, déconstruit, dont seraient arbitrairement dissociés les composants actifs, si l'on ne s'avisait tout de même que les choses in vivo se passent autrement.

Si Norton \& Sackville ont choisi de placer leurs tableaux vivants avant et non après l'acte parlé, c'est bien pour assurer malgré tout le fonctionnement du troisième renvoi référentiel, ainsi mémorisé et plus ou moins consciemment et efficacement juxtaposé aux deux autres durant le déroulement de la partie B. Tout semble donc se passer comme si la séquence $A+B$ assurait le plein et entier fonctionnement de la diaphore, mais dans la successivité. Et là réside peut-être l'effet recherché par les auteurs de Gorboduc.

En réservant en effet à chacun des deux aspects du texte un moment de fonctionnement propre au sein du procès dramatique, et en affectant prioritairement à chacun l'une des modalités - la spatiale puis la temporelle - de la réalisation théâtrale, il semble que les dramaturges aient voulu exploiter et contrôler à la fois au mieux l'éclatant pouvoir des vertus de l'image et des forces du verbe. En dissociant la pensée discursive, tissée de durée et de lenteur et surtout accordée à l'âme intellective, d'un autre système de signes (ou complexe de systèmes) qui adresse d'abord ses sollicitations à l'œil et à l'oreille, le créateur de cette forme éclatée procède moins peut-être à 
un divorce mutilant qu'à la libération des virtualités successivement déployées par chacun des deux processus formels. Les deux parties sollicitées dans le spectateur - Fancy et Wit-peuvent, consécutivement touchées, pleinement occuper le moment qui leur est dévolu. De même que la diérèse permet le plein épanouissement de chacun des deux éléments vocaliques, de même l'impact des représentations sensibles puis conceptuelles atteindraient ainsi leur plénitude.

L'hypothèse ne paraîtra peut-être pas invraisemblable à qui sait la fascination de l'époque pour l'image, créditée d'inquiétants pouvoirs, et son appétit pour le verbe musical, rhapsodique, lyrique ou rhétorique, ni à qui considère l'intensité sémantique qui habite volontiers l'une et l'autre des deux formes.

Pourtant, cette idée ne restitue que partie de l'ambiguïté de Gorboduc. Il faut, me semble-t-il, la doubler de l'hypothèse contraire pour obtenir, dans la complémentaire antinomie des deux, un schéma explicatif satisfaisant. On ne peut en effet pas congédier aisément le jugement objectivement fondé que la pièce s'offre dans une forme compassée, d'une artificialité un peu inhumaine, et s'il est un moyen de rendre compte de la violence potentielle de son discours, iconique ou verbal, c'est de considérer que ce feu sous la glace serait le produit d'un soigneux équilibre. Selon cette vue, la rhétorique corsetée de Gorboduc, la brutalité allégoriquement esquissée dans ses tableaux seraient l'expression mesurée et réduite d'une violence contenue. Dans cette pièce, comme elle le fait dans le corps alchimique, couverait l'explosive brièveté du sens.

Le non-spécialiste (que je suis) embarqué dans l'hypothèse, ne peut formuler que de façon interrogative les raisons suceptibles de lui donner créance. J'imagine au moins trois séries de mobiles derrière ce que j'ai lu comme une stratégie théâtrale :

- le respect très conscient des vues humanistes touchant aux procédures de la formalisation esthétique: ainsi, la diégèse néoplatonicienne ou la fable aristotélicienne ont-elles préséance sur la mimésis plus vulgaire. Cette explication rend pourtant mal compte, à elle seule, des procédures retenues.

- la précaution, politique et quasiment déontologique chez nos auteurs, de ne pas abandonner à l'imagination vulgaire l'utilisation inconsidérée du plein sens. L'absence, contraire à l'usage populaire, de glose explicative des tableaux suppose que la réassociation de leur 
message avec celui du texte écrit se fasse dans le ressouvenir et la réflexion. Comme on éviterait le contact explosif de produits hautement réactifs en les isolant l'un de l'autre, ainsi les deux formalisations étanches de Gorboduc garantiraient-elles que leur réassociation, réalisée dans la durée, resterait accessible à l'esprit de réflexion et de contemplation, non à la fougueuse et plébéienne émotion sensible.

- enfin (mais aucune de ces hypothèses ne paraît vraiment exclusive des autres) n'est-ce pas aussi chez nos aristocratiques auteurs le choix d'une esthétique raffinée préférant, pour une sensibilité exigeante qui trouve plaisir à la contention et la discrétion, les structures de la réduction et du discontinu?

Tant de questions débouchent sur l'ultime interrogation que pose depuis le début cette forme théâtrale à tous égards rare : une production spectaculaire fondée sur la dissociation, afin de réserver l'intensité de son sens à la contemplation intellectuelle, trouve-t-elle vraiment à son époque le destinataire idéal rompu au déchiffrage qu'elle réclame; ou bien doit-on considérer à l'inverse qu'elle est déjà expérience anormale, sinon aberrante, en voie de rompre avec les processus essentiels de la communication théâtrale?

Cette question est au vrai celle de la pertinence culturelle d'une pièce qui, explorant effectivement la voie d'une dissociation théâtrale, et suscitant une perception filée et étirée jusqu'à la stase contemplative, s'aventure vers une forme de théâtre intimisé qui ne constitue peut-être plus la voie royale de l'expression théâtrale.

\section{CONCLUSION}

Cet examen visait à repérer, au sein d'œuvres que l'on hésite à classer comme de francs succès de la production théâtrale, deux traits corrélés, à travers l'usage qu'elles font du lieu et du temps dramatiques :

- leur cohérence esthétique ;

- la visée fonctionnelle que paraît vouloir servir leur architecture.

Dans ces deux formes apparemment antinomiques, à deux moments de la culture et dans des genres dramatiques différents, j'ai cru déceler des intentions similaires en ce que toutes deux, explorant 
le principe fondateur du fonctionnement tréâtral, le trouble référentiel, portent la forme même dont elles sont issues jusqu'au bord de la rupture.

Ce faisant, et à l'inverse, cette exploration et la distortion qui en résulte dévoilent deux parti-pris qui sont de sens sociologique autant que théâtral, et où, en se gardant de trop tirer d'une comparaison unique, on peut voir la marque de deux moments différents de l'évolution culturelle.

Chez Medwall, la subversion de la forme, y compris la formalisation spatio-temporelle, procède d'un souci d'inclusivité généralisée. Ceci est sans doute une recette théâtrale efficace, reprise et mise au goût du jour dans certaines concoctions comiques élizabéthaines, mais c'est aussi l'index de l'indéniable et bien sûr relatif œcuménisme culturel qui la licite.

Chez nos juristes élizabéthains par contre, le refus de l'effusion théâtrale, enserrée dans le rigoureux codage de la pensée et de l'émotion, paraît attester, d'une part la proximité de la césure qui va de plus en plus différencier les destinataires d'une même forme. De l'autre, il manifeste l'esprit nouveau, celui de la modernité, dans la mesure où la restitution de l'espace-temps théâtral rompt avec la stricte forme physique du monde et génère une reconstruction qui est sans doute de fonction encore didactique mais dont le caractère symbolique est déjà largement asservi à des fins esthétiques.

André LASCOMBES

Université de Tours

\section{NOTES}

1) Les références sont à l'ouvrage de Anne Righter, Shakespeare and the Idea of the Play dans l'édition Penguin, Penguin Shakespeare Library, 1967.

2) Pour un exposé ordonné de cette question, même avec la restriction faite, voir A. Ubersfeld, L'Ecole du spectateur, Paris : Ed. Sociales, 1981, chap.1.

3) A. Lascombes, Culture et théâtre populaire en Angleterre à la fin du Moyen Age, thèse d'Etat, Paris III, 1980, chap. 8 ; et " Du masque au visage : diaphore théâtrale et typologie du personnage dans les Cýcles anglais ", Atti del IV Colloquio della S.I.E.T.M., Viterbo, 1984, 349-62. 
4) A. Righter, op. cit.,, 31-40.

5) G. Wickham ed. English Moral Interludes, London : Dent, 1976, 39.

6) R. Southern, The Staging of Plays Before Shakespeare, London: Faber \& Faber, 1973, passim, évalue les possibles fonctions scéniques de ce côté du hall Tudor.

7) Southern, op. cit., 96-97.

8) La réduction du temps de la fiction primaire au temps de la représentation s'opère en plusieurs fois par le biais de: - contractions événementielles (vers 292-302 et 494-97; 653-84) ; - accélérations de la chronologie initialement proposée pour la fiction, et qui passe de quelques jours (342 et 565) à « un jour ou deux" (459; 567-8) et enfin à "ce jour-même ) (1293). Ces deux opérations interviennent au cours de la première partie, à laquelle renvoie la numérotation des vers cités.

9) Voir en particulier les passages suivants: $1^{\mathrm{e}}$ partie, $1-201 ; 2^{\mathrm{e}}$ partie, 1-74; $76-105 ; 131-165 ; 874-78$.

10) Les références sont à l'édition de la pièce dans le recueil Minor Elizabethan Drama, London : Dent (Everyman's Lib.), 1964, 1-53.

11) R. Southern, op. cit., 473-4.

12) W. Clemen, English Tragedy Before Shakespeare, London: Methuen, 1961, 57 ; Paul Bacquet, "Structure et valeur dramatiques de Gorboduc ", Filološki pregled (1964) 1, 247-59.

13) D. Mehl, The Elizabethan Dumb show, London : Methuen, 1965, 30-41.

14) Les seules autres évocations de la réalité topographique du lieu de la fiction seraient à chercher dans les hâtives esquisses dues à Eubulus (I, 2, 333-4 et 396-9) et à Dordan (II, 1, 36-44). Dans ce dernier cas, on reste très loin des développements imaginatifs dus au Gaunt shakespearien (Richard II, II, 1, 40-68), voire à certaines des sources de ce passage, dont The Mirror for Magistrates. Voir Peter Ure in Richard II ed. The Arden Shekespeare, 50.

15) IV, 1, 279-347. Voir encore, dans la même scène, les vers $82-9,161,168-9,185-6$, 219-23. 